\title{
Assembling curvature continuous surfaces from triangular patches
}

\author{
K. Karčiauskas and J. Peters \\ Vilnius University, University of Florida
}

\begin{abstract}
We assemble triangular patches of total degree at most eight to form a curvature continuous surface. The construction illustrates how separation of local shape from representation and formal continuity yields an effective construction paradigm in partly underconstrained scenarios. The approach localizes the technical challenges and applies the spline approach, i.e. keeping the degree fixed but increasing the number of pieces, to deal with increased complexity when many patches join at a central point.
\end{abstract}

Key words: $C^{2}$ surface construction, shape, guide surface, triangular patches

\section{Introduction}

Complex surface blends, for example when capping a $C^{2}$ spline surface by $n$ patches, require an increase in either the degree or the number of pieces compared to the surrounding regular spline surface. Typically, the new degrees of freedom do not match the formal continuity constraints and this results in an underconstrained problem. One way to set the extra degrees of freedom is to minimize a geometrically-motivated functional, say approximating an integral of the mean, Gauss or total curvature (see e.g. [12, 20, 4]; these functionals have also been applied to faceted representations - but here we are only concerned with curvature continuous surfaces). Another is to minimize deviation from a space that has too few degrees of freedom, for example by minimizing a quadratic expression of (a convex combination of) higher-order derivatives and hence penalizing higher degree (cf. Definition 1 and Figure 13). We use a third approach to setting extra degrees of freedom: we first create a surface fragment that captures the shape and then approximate this surface fragment to satisfy the smoothness constraints. This two-stage approach of separating shape from formal smoothness constraints was introduced by Karčiauskas and Peters in [7]; and was already hinted at by the composition with quadratic shapes in [15] and [19]. We call it guided surfacing in the following. Guided surfacing according to [7] has the nice side-effect of localizing, otherwise global, smoothness constraints. This very much simplifies derivation and analysis and avoids the need to invert large matrices during construction.

There is a rich literature on construction of $C^{2}$ surfaces based on quadrilateral meshes and patches, e.g. $[6,2,15,21,19,3$, $10,11,13,5,7,8]$. Here, we consider three-sided patches as in [17], corresponding to control nets with triangular facets. Since box-splines do a good job when those meshes are regular, i.e. all vertices have the same valence 6 , the challenge is to complete a $C^{2}$ box-spline surface by filling its isolated multi-sided holes (see Figure 1, right) using $n$ (macro-)patches for an $n$ sided hole. Our surface construction below is interesting in its own right since the degree of the guided macro-patches output
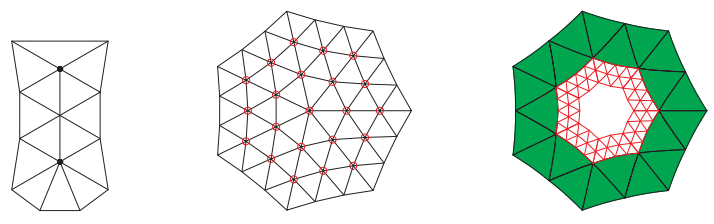

Figure 1: Input triangulation and boundary data. (left) No two extraordinary vertices are direct neighbors. (middle) Triangulation with a single, isolated central non-6-valent vertex, called extraordinary vertex. We interpret the vertices as box-spline coefficients that define the (green) piecewise degree 4 surface ring right. (This ring is not used in the construction, but will allow us to check the quality of the multi-sided blend to existing data.) The circled vertices (middle) define the boundary data $\mathbf{b}$ : position, first and second derivative, for extending the surface ring. (right) These boundary data are shown as a depth three (red) net of a Bernstein-Bézier (BB) coefficients.

is 8 , the same as the lowest total-degree surface constructions in the literature $[14,17,18]$ (a bound also for finite element spaces [9, Ex 5.1]), but has better shape control. Our main aim, however, is to illustrate that separating shape from smoothness constraints allows setting unconstrained parameters in a natural way. We show how a high number $n$ of features such as in high-order saddles, can be made to blend with slowly dissipating curvature differences, not by increasing the degree of the surface, but the number of polynomial pieces in each patch and by using the underlying guide surface to set unconstrained parameters (such as the $\mathbf{a}_{i j}$ in the local construction on page 4) in a geometrically intuitive fashion, by sampling.

Concretely, we consider the following setup. We are given a triangulation with isolated extraordinary vertices, i.e. vertices of valence $n \neq 6$ such that each direct neighbor is regular of valence 6 (Figure 1, middle). That is, every triangle has at least two regular vertices. Wherever a triangle has three regular vertices, we interpret the vertices of the triangle and their neighbor vertices as (box-spline) control points of a three-sided polynomial patch of total degree 4 , defined by the three-direction $C^{2}$ box-spline [1] with directions $\Xi:=\left[\begin{array}{cccccc}1 & -1 & 0 & 0 & 1 & -1 \\ 0 & 0 & 1 & -1 & 1 & -1\end{array}\right]$. Each extraordinary vertex of valence $n$ in the triangular mesh causes an $n$-sided hole in the regular surface complex. Assuming that 
such vertices are separated (Figure 1, left), we want to fill each hole with a cap consisting of $n$ macro-patches of degree 8 so that the resulting surface is

- curvature continuous,

- the geometry of the cap incorporates that of the surrounding surface, and

- the cap does not fluctuate in position, normal or curvature.
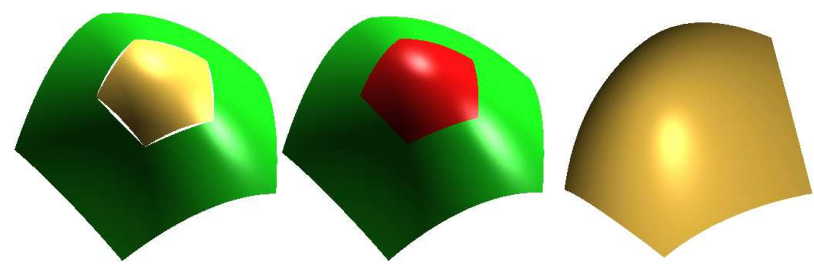

Figure 2: Guided surfacing. (left) A $C^{2}$ guide $\mathbf{g}$ (yellow cap) is determined with reference to the boundary data $\mathbf{b}$ defined by a surrounding (green) surface. The guide surface and the surrounding surface are in general not even connected (see Section 2 and the Appendix for the derivation of a guide with the control net structure of Figure 3, bottom,left). (middle and right) the final piecewise polynomial $C^{2}$ surface without gap: the algorithm to be specified constructs the red replacement of the guide that matches the boundary data $C^{2}$ after reparameterization.

Construction overview. Our approach is as follows. First, we construct a piecewise $C^{2}$ guide surface piece $\mathbf{g}: \mathbb{R}^{2} \rightarrow \mathbb{R}^{3}$ (Figure 2, left). This surface represents the design intent in the sense that the final surface will follow its shape. Even when the guide takes into account the boundary data $\mathbf{b}$, it is typically not suitable for a final cap $\mathbf{x}$ since, as illustrated in Figure 2, left ${ }^{1}$, it need not even join continuously with the surrounding surface and can have a completely different representation from the one needed for further processing. We define a $C^{2}$ reparameterization $\rho: \mathbb{R}^{2} \rightarrow \mathbb{R}^{2}$ from the boundary of the domain of $\mathbf{x}$, where always six patches meet at a vertex, to the center, where $n \neq 6$ patches meet; and an operator $h$ that takes as input a sufficiently smooth function and constructs surface pieces of degree 8 from the derivatives of its input. Then the cap completing a $C^{2}$ surface is defined by $\mathbf{x}:=h(\mathbf{g} \circ \boldsymbol{\rho})$.

Paper Overview. In Section 2, we construct the $C^{2}$ map $\rho$ as well as a prototype guide $\mathbf{g}$, whose instantiation, in dependence of the boundary data $\mathbf{b}$ is described in the Appendix. In Section 3, we construct $h$ piecemeal, via a simple local operator $h_{\diamond}^{8}$ acting only at vertices of $\mathbf{g} \circ \boldsymbol{\rho}$. The surface construction then becomes a straightforward localized enforcement of $C^{2}$ constraints. In Section 4, we apply the tools of Section 3 to obtain a surface cap that completes a $C^{2}$ surface (as in Figure 2) and has good shape for $n<9$. In Section 5, we address the cases $n \geq 9$ by modeling each of the $n$ segments of the cap by four polynomial pieces. In Section 6, we discuss modifications to obtain good shape both for very high and very low valences; and we illustrate how derivative-based functionals applied in $\mathbb{R}^{3}$ fail to achieve the same effect as guide surfaces.

${ }^{1}$ This paper is concerned with shape and curvature continuity up close. Therefore it makes little sense to show any large triangulations where shape would be dominated by regular box-splines. The figures in the submission can be enlarged using the pdf zoom capability.
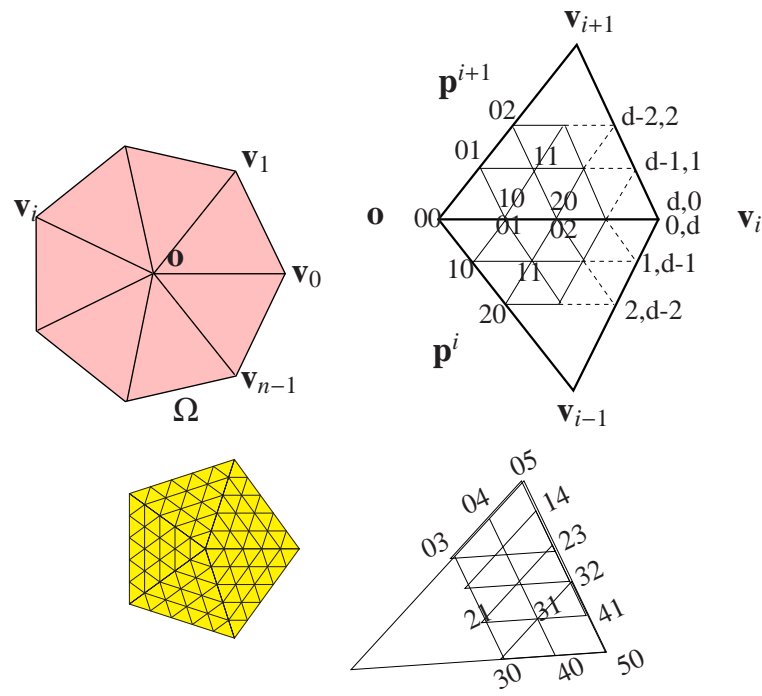

Figure 3: Domain and Indexing. (top,left) 7-sided domain $\Omega(n=7)$. (top, right) BB coefficient indices near the common boundary of an adjacent pair of polynomials $\mathbf{p}^{i}$ and $\mathbf{p}^{i+1}$. (bottom, left) BB control net of a piecewise degree 5 guide with $n=5$ pieces. (right) Indices of one segment of degree 5 .

\section{2. $C^{2}$ functions on a polygonal domain}

In this section, we prepare the technical background to be able to focus later only on the high-level construction. We define an $n$-gon $\Omega \subsetneq \mathbb{R}^{2}$, a $C^{2}$ map $\rho$ that maps $n$ copies of the right angle unit triangle $\Delta$ to $\Omega$ and the guide $\mathbf{g}$ that maps $\Omega$ to $\mathbb{R}^{3}$. Also the final hole-filling spline cap $\mathbf{x}$ maps $\Delta \times\{1, \ldots, n\} \rightarrow \mathbb{R}^{3}$; but it will only be discussed in later sections. Given the purpose of each map, its smoothness and symmetry, the derivations use standard machinery in geometric design. But this does not mean that the derivation is trivial. It reflects important choices of properties and polynomial degree. Until Section 6, we assume

$$
n \notin\{3,4,6\}
$$

since $n=6$ corresponds to the box-spline construction and $n=$ 3 and $n=4$ admit special (simpler) treatment, explained in Section 6.

\subsection{The $n$-sided domain $\Omega$}

The $n$-gon $\Omega$ is composed of $n$ triangles

$\triangle \mathbf{o v}_{i} \mathbf{v}_{i+1}:=\left\{\mathbf{o}+u\left(\mathbf{v}_{i}-\mathbf{o}\right)+v\left(\mathbf{v}_{i+1}-\mathbf{o}\right), 0 \leq u, 0 \leq v, u+v \leq 1\right\}$,

where $i=0 \ldots n-1$ and, as illustrated in Figure 3, top right,

$$
\Omega:=\bigcup \triangle \mathbf{o v}_{i} \mathbf{v}_{i+1}, \quad \mathbf{o}:=\left[\begin{array}{l}
0 \\
0
\end{array}\right], \mathbf{v}_{i}:=\left[\begin{array}{c}
\cos (i \alpha) \\
\sin (i \alpha)
\end{array}\right], \alpha:=\frac{2 \pi}{n} .
$$

The image of $\Delta \mathbf{o v}_{i} \mathbf{v}_{i+1}$ will be the $i$ th segment of the map to be constructed. We also define the unit triangle $\Delta \in \mathbb{R}^{2}$ and $\angle \mathbf{v}_{0} \mathbf{v}_{1}$, a sector of the plane bounded by the rays $\mathbf{o v}_{0}$ and $\mathbf{o v}_{1}$. With the Bernstein-Bézier (BB) coefficients $\mathbf{p}_{j k}^{i}$ (see e.g. [16, Ch 10]) indexed as in Figure 3, top right, the well-known conditions for 
two patches $\mathbf{p}^{i}$ and $\mathbf{p}^{i+1}$ abutting along the boundary defined by $\mathbf{p}_{j 0}^{i+1}=\mathbf{p}_{0 j}^{i}$ to join (parametrically) $C^{1}$, respectively $C^{2}$ are

$$
\begin{aligned}
\mathbf{p}_{j 1}^{i+1}= & v_{0} \mathbf{p}_{0 j}^{i}+v_{1} \mathbf{p}_{0, j+1}^{i}+v_{2} \mathbf{p}_{1 j}^{i}, \\
\mathbf{p}_{j 2}^{i+1}= & v_{0}^{2} \mathbf{p}_{0 j}^{i}+2 v_{0} v_{1} \mathbf{p}_{0, j+1}^{i}+v_{1}^{2} \mathbf{p}_{0, j+2}^{i} \\
& +2 v_{0} v_{2} \mathbf{p}_{1 j}^{i}+2 v_{1} v_{2} \mathbf{p}_{1, j+1}^{i}+v_{2}^{2} \mathbf{p}_{2 j}^{i},
\end{aligned}
$$

with scalar weights determined by $\mathbf{v}_{i+1}=v_{0} \mathbf{o}+v_{1} \mathbf{v}_{i}+v_{2} \mathbf{v}_{i-1}$, as

$$
v_{0}:=2 \overline{\mathrm{c}}, v_{1}:=2 \mathrm{c}, v_{2}:=-1, \quad \mathrm{c}:=\cos \frac{2 \pi}{n}, \overline{\mathrm{c}}:=1-\mathrm{c} .
$$

\subsection{A parametrization of $\Omega$}

The $C^{2}$ parametrization $\rho$ consists of $n$ copies, rotated by $i \alpha$, of a polynomial piece $\rho: \Delta \rightarrow \angle \mathbf{v}_{0} \mathbf{v}_{1} \subsetneq \mathbb{R}^{2}$. The image of $\rho$ (see Figure 4) does not exactly match the domain $\Omega$ of the guide $\mathbf{g}$, but that is not a problem since the pieces of $\mathbf{g}$ are polynomial and well-defined on the whole sector.

We start by defining three layers of BB-coefficients $\gamma_{i j}, i+j>$ 3 , of a polynomial $\gamma: \Delta \rightarrow \Delta$ of total degree 5. Composition with this map will allow for a $C^{2}$ transition across the sector partitioning lines and define a $G^{2}$ transition from the cap to the surrounding surface ring. Since the map is symmetric with respect to exchange of coordinates, the layers are defined by

$$
\begin{aligned}
\gamma_{5-j, j} & :=\frac{1}{5}\left[\begin{array}{c}
5-j \\
j
\end{array}\right], \quad j=0, \ldots, 5, \\
\gamma_{40} & :=\left[1-\frac{1}{10 \bar{c}}, 0\right]^{\mathrm{t}}, \gamma_{31}:=\left[\frac{14 \overline{\mathrm{c}}-1}{20 \overline{\mathrm{c}}}, \frac{1}{5}\right]^{\mathrm{t}}, \\
\gamma_{22} & :=\frac{26 \overline{\mathrm{c}}-1}{60 \overline{\mathrm{c}}}[1,1]^{\mathrm{t}}, \gamma_{30}:=\left[\frac{15 \overline{\mathrm{c}}^{2}-4 \mathrm{c} \overline{\mathrm{c}}+\mathrm{c}^{2}}{20 \overline{\mathrm{c}}^{2}}, 0\right]^{\mathrm{t}}, \\
\gamma_{21} & :=\left[\frac{14 \overline{\mathrm{c}}-1}{30 \overline{\mathrm{c}}}+\frac{r}{20}, \frac{24 \overline{\mathrm{c}}^{2}+\overline{\mathrm{c}}-\mathrm{c}}{120 \overline{\mathrm{c}}^{2}}\right]^{\mathrm{t}} .
\end{aligned}
$$

An optimization explained below determines the scalar parameter $r$ for valences $n=3, \ldots, 12$ (6 being regular) as

$$
\begin{array}{cccccc}
n= & 3 & 4 & 5 & 7 & 8 \\
r:= & 1.06044 & 0.7 & 0.28139 & 0.18307 & 1.10725 \\
& & 9 & 10 & 11 & 12 \\
& & 0.42692 & 1.02770 & 2.02745 & 3.54287
\end{array} .
$$

The map $\gamma$ was carefully chosen so that along the outer edge

$$
u+v=1: \quad \operatorname{deg} \gamma=1, \quad \operatorname{deg} \partial_{v} \gamma=2, \quad \operatorname{deg} \partial_{v}^{2} \gamma=3,
$$

and hence the expansion up to order 2 of the composition with a map of total degree 4 (such as the boundary data, or the map $\chi$ below) is of total degree at most 8 along the outer edge.

The polynomial piece $\rho$ is of degree 8, symmetric with respect to the bisector of the sector $\Omega$ (Figure 4) and connects $C^{2}$ to its rotated copy. It will be composed with the guide to construct the final surface. The outermost three layers of coefficients $\rho_{i j}, i+j>5$, are those of one segment of the characteristic ring of Loop subdivision, $\chi: \Delta-\frac{\Delta}{2} \rightarrow \angle \mathbf{v}_{0} \mathbf{v}_{1} \subsetneq \mathbb{R}^{2}$, composed with $\gamma: \rho:=\chi \circ \gamma$. The layers form a single polynomial piece since, at the outer edge $u+v=1, \chi$ is the $C^{2}$ prolongation of one polynomial piece (of a scaled copy of $\chi$ ). Since the outer

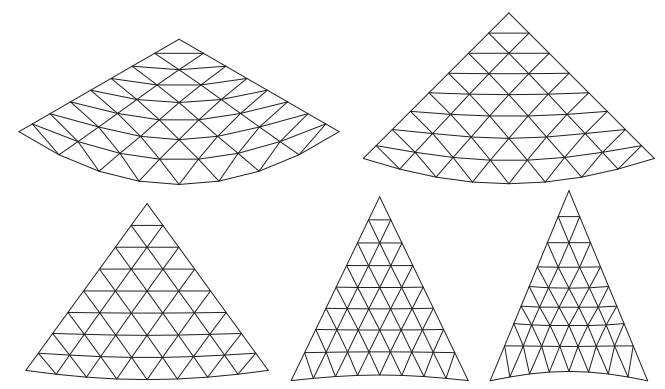

Figure 4: BB control net of the bivariate reparametrization $\rho$ (rotated by $-(\pi+$ $\alpha) / 2$ ) for $n=3,4,5,7,8$.

boundary of $\gamma$ is linear, the outer boundary of $\rho$ is that of $\chi$. For $i+j \leq 5$, enforcing bisector symmetry as well as $C^{2}$ continuity between adjacent rotated pieces of $\rho$ leaves only five degrees of freedom. Together with $r$ of $\gamma$, these 6 scalars are determined by minimizing a functional $\mathcal{F}_{4}(\rho)$.

Definition 1. For a sufficiently smooth function $f(u, v)$ defined over some triangle $\triangle$,

$$
\mathcal{F}_{m}(f):=\int_{\Delta} \sum_{i+j+k=m}\left(\begin{array}{c}
m \\
i j k
\end{array}\right)\left(\partial_{u^{i} v^{j}(u-v)^{k}}^{m} f\right)^{2},(i, j, k \geq 0) .
$$

For a map $f:=\left(f_{1}, f_{2}\right): \Delta \rightarrow \mathbb{R}^{2}, \mathcal{F}_{m}(f):=\mathcal{F}_{m}\left(f_{1}\right)+\mathcal{F}_{m}\left(f_{2}\right)$.

Note that we apply $\mathcal{F}_{4}(\rho)$ only to reparameterizations $\mathbb{R}^{2} \rightarrow \mathbb{R}^{2}$, not to surfaces embedded in $\mathbb{R}^{3}$ where use of $\mathcal{F}_{m}$ is questionable (see the example of Figure 13).

\subsection{A $C^{2}$ spline on $\Omega$}

In preparation for a construction of the guide surface (in the Appendix), we construct a $C^{2}$ spline

$$
\mathbf{g}: \Omega \subsetneq \mathbb{R}^{2} \mapsto \mathbb{R}^{3}
$$

In each sector, $\mathbf{g}$ is represented in total degree, triangular Bézier form with coefficients $\mathbf{g}_{j k}^{i}$ as shown in Figure 5. The smoothness conditions (3) and (4) imply

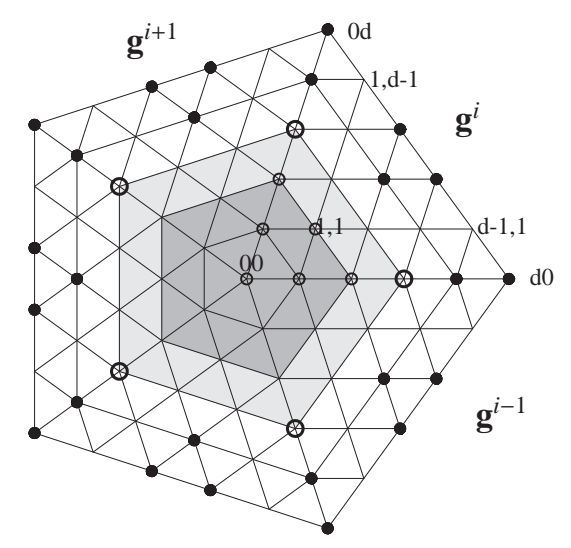

Figure 5: BB-control net structure of the $C^{2}$ geometry map g of degree $d$. 
(a) (darker gray region in Figure 5.) the extraordinary point $\mathbf{g}_{00}^{i}$ and its five nearest coefficients $\mathbf{g}_{10}^{i}, \mathbf{g}_{01}^{i}, \mathbf{g}_{20}^{i}, \mathbf{g}_{11}^{i}, \mathbf{g}_{02}^{i}$ define the same quadratic polynomial (in degree-raised BBform) for all $i$;

(b) (light gray region in Figure 5) the coefficients $\mathbf{g}_{30}^{i}$ can be chosen freely. If the coefficients $\mathbf{g}_{21}^{i}$ satisfy the circulant system

$$
\begin{aligned}
& \mathbf{g}_{21}^{i-1}+4 \mathrm{cg}_{21}^{i}+\mathbf{g}_{21}^{i+1}=2 R_{i}, \\
& R_{i}:=2 \mathrm{c}^{2} \mathbf{g}_{30}^{i}+\mathrm{cg}_{03}^{i}+4 \mathrm{c} \overline{\mathbf{c}} \mathbf{g}_{20}^{i}-2 \overline{\mathrm{c}} \mathbf{g}_{11}^{i}+\overline{\mathrm{c}} \mathbf{g}_{02}^{i}+2 \overline{\mathrm{c}}^{2} \mathbf{g}_{10}^{i},
\end{aligned}
$$

then setting the coefficients $\mathbf{g}_{12}^{i}$ according to (3) enforces the $C^{2}$ constraints.

The Appendix shows one option for setting the remaining degrees of freedom, e.g. $\mathbf{g}_{00}^{0}, \mathbf{g}_{10}^{0}, \mathbf{g}_{01}^{0}, \mathbf{g}_{20}^{0}, \mathbf{g}_{11}^{0}, \mathbf{g}_{02}^{0}$ in (a).

By applying the discrete Fourier transform (DFT), solving the transformed system and then applying the inverse DFT, we obtain the following solution to system (8).

Lemma 1. For $n \geq 5, n \neq 6$, the unique solution of system (8) is

$$
\mathbf{g}_{21}^{i}=\frac{1}{n} \sum_{l=0}^{n-1} \sum_{j=0}^{n-1} \frac{R_{l} \cos ((i-l) j \alpha)}{2 \mathrm{c}+\cos (j \alpha)} .
$$

\section{Reduction to a localized $C^{2}$ transition between two patches}

An operator $h^{8}$ that constructs a new polynomial patch $\mathbf{p}$ of degree 8 from a given patch $\mathbf{f}$, such as $\mathbf{g} \circ \boldsymbol{\rho}$, was defined in [7]. The operator samples partial derivatives $\left(\partial_{u}^{l} \partial_{v}^{j} \mathbf{f}\right)_{l+j<5}$ and $\partial_{u}^{2} \partial_{v}^{3} \mathbf{f}, \partial_{u}^{3} \partial_{v}^{2} \mathbf{f}$ at a point (dark grey in Figure 6, left), and converts these derivatives into the coefficients of a BB-patch of degree 8 . The coefficients from all three corners of a triangle determine a patch of total degree 8 over the triangle. We use a slightly sim-

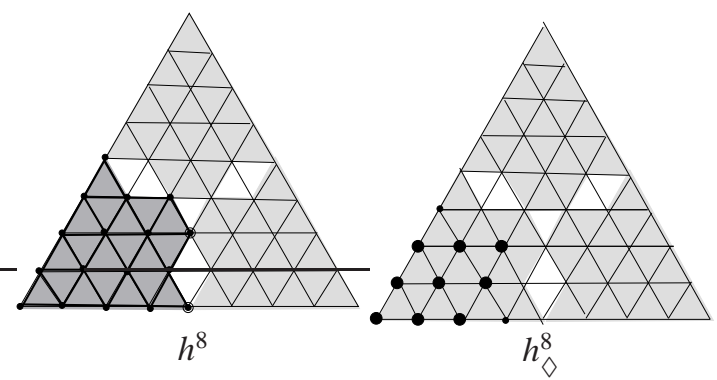

Figure 6: Localizing via sampling. (left) The operator $h^{8}$ from [7] constructs a patch of degree 8 from partial derivatives. (right) Operator $h_{\diamond}^{8}$ returns a subset of coefficients (of one, two or three corners), here indicated for one corner by $\bullet$.

pler operator $h_{\diamond}^{8}$ that returns only the BB-coefficients marked as bullets in Figure 6, right. When $h_{\diamond}^{8}$ is applied at the corners (with their respective endpoints' valences) of two $C^{2}$-connected patches $\mathbf{f}$ and $\tilde{\mathbf{f}}$, the resulting BB-coefficients (Figure 7) define $2 \times 15$ corner coefficients of adjacent surface pieces $\mathbf{p}$ and $\tilde{\mathbf{p}}$ (see Figure 7). Since the derivatives are sampled from a $C^{2}$ surface, $\mathbf{f}, \tilde{\mathbf{f}}$, this construction guarantees $C^{2}$ continuity of $\mathbf{p}, \tilde{\mathbf{p}}$ at the two ends of the shared curve; and it localizes the task of enforcing the remaining $C^{1}$ and $C^{2}$ constraints between segments and across the boundary of $\Omega$. To emphasize this locality,

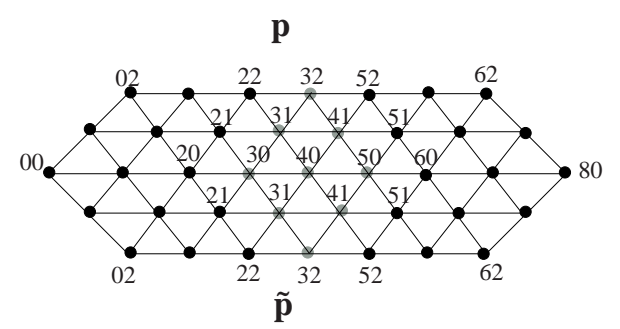

Figure 7: Local construction and symmetric indexing of the coefficients near the boundary of two patches $\mathbf{p}$ and $\tilde{\mathbf{p}}$. The BB-coefficients determined by applying $h_{\diamond}^{8}$ at the corners of two adjacent patches are indicated by $\bullet$. The coefficient 30 is implied by smoothness.

we rewrite (3) and (4) with symmetric indexing as in Figure 7, right. For a patch $\mathbf{p}$ with domain vertices $\mathbf{w}_{0}, \mathbf{w}_{1}, \mathbf{w}_{2}$ and a patch $\tilde{\mathbf{p}}$ with domain $\mathbf{w}_{0}, \mathbf{w}_{2}, v_{0} \mathbf{w}_{0}+v_{1} \mathbf{w}_{1}+v_{2} \mathbf{w}_{2}$, the $C^{1}$ and $C^{2}$ constraints (3) and (4) become respectively

$$
\begin{aligned}
\tilde{\mathbf{p}}_{j 1}= & v_{0} \mathbf{p}_{j 0}+v_{1} \mathbf{p}_{j+1,0}+v_{2} \mathbf{p}_{j 1} \\
\tilde{\mathbf{p}}_{j 2}= & v_{0}^{2} \mathbf{p}_{j 0}+2 v_{0} v_{1} \mathbf{p}_{j+1,0}+v_{1}^{2} \mathbf{p}_{j+2,0} \\
& +2 v_{0} v_{2} \mathbf{p}_{j 1}+2 v_{1} v_{2} \mathbf{p}_{j+1,1}+v_{2}^{2} \mathbf{p}_{j 2}
\end{aligned}
$$

Setting (note $v_{i} \neq 0$ by our valence assumption)

$$
\mathbf{p}_{30}:=\left(-v_{0} \mathbf{p}_{20}-v_{2} \mathbf{p}_{21}+\tilde{\mathbf{p}}_{21}\right) / v_{1}
$$

and, symmetrically, $\mathbf{p}_{50}:=\left(-v_{1} \mathbf{p}_{60}-v_{2} \mathbf{p}_{51}+\tilde{\mathbf{p}}_{51}\right) / v_{0}$ is then equivalent to extending $h_{\diamond}^{8}$ by $\partial_{u}^{3} \mathbf{f}$ and therefore enforces simultaneously the constraint (9) for $j=2$ and (10) for $j=1$. (Alternatively, we could have increased the operator $h_{\diamond}^{8}$ by $\partial_{u}^{3} \mathbf{f}$ and $\partial_{v}^{3} \mathbf{f}$, i.e. used more of the output of $h^{8}$, but we want to emphasize the locality of the construction.) Of the remaining 7 coefficients, two can be set freely when enforcing $C^{2}$ continuity. We set $\mathbf{p}_{40}:=\mathbf{a}_{40}$, a point to be derived from the guide surface (see Section 4) and determine $\mathbf{p}_{32}$ and $\tilde{\mathbf{p}}_{32}$ to minimize $\left\|\mathbf{p}_{32}-\mathbf{a}_{32}\right\|^{2}+\left\|\tilde{\mathbf{p}}_{32}-\tilde{\mathbf{a}}_{32}\right\|^{2}$ for points $\mathbf{a}_{32}, \tilde{\mathbf{a}}_{32}$ derived from the guide surface. Then (9) and (10) are enforced by setting

$$
\begin{aligned}
\mathbf{p}_{31}:= & \frac{1}{2 v_{1} v_{2}}\left(v_{0}^{2} \mathbf{p}_{20}-v_{1}^{2} \mathbf{p}_{40}-v_{2}^{2} \mathbf{p}_{22}+\tilde{\mathbf{p}}_{22}-2 v_{0} \tilde{\mathbf{p}}_{21}\right) \\
\mathbf{p}_{32}:= & \alpha_{32} \mathbf{a}_{32}+\tilde{\alpha}_{32} \tilde{\mathbf{a}}_{32}+\alpha_{21} \mathbf{p}_{21}+\alpha_{51} \mathbf{p}_{51}+\alpha_{22} \mathbf{p}_{22}+\alpha_{42} \mathbf{p}_{42} \\
& +\tilde{\alpha}_{21} \tilde{\mathbf{p}}_{21}+\tilde{\alpha}_{51} \tilde{\mathbf{p}}_{51}+\tilde{\alpha}_{22} \tilde{\mathbf{p}}_{22}+\tilde{\alpha}_{42} \tilde{\mathbf{p}}_{42} \\
\alpha_{32}:= & \frac{1}{1+v_{2}^{4}}, \tilde{\alpha}_{32}:=\frac{v_{2}^{2}}{1+v_{2}^{4}}, \\
\alpha_{21}:= & \frac{v_{0}^{2} v_{2}^{3}}{v_{1}\left(1+v_{2}^{4}\right)}, \tilde{\alpha}_{21}:=\frac{\alpha_{21}}{v_{2}}, \alpha_{22}:=\frac{v_{0} v_{2}^{4}}{v_{1}\left(1+v_{2}^{4}\right)}, \tilde{\alpha}_{22}:=-\frac{\alpha_{22}}{v_{2}^{2}}
\end{aligned}
$$

By combinatorial symmetry and abbreviating $\left[\tilde{v}_{0}, \tilde{v}_{1}, \tilde{v}_{2}\right]:=$ $\left[-v_{0},-v_{1}, 1\right] / v_{2}$, 


\begin{tabular}{l|l|l} 
we obtain & from & by exchanging $(\leftrightarrow)$ \\
\hline$\alpha_{51}, \alpha_{42}, \tilde{\alpha}_{51}, \tilde{\alpha}_{42}$ & $\alpha_{21}, \alpha_{22}, \tilde{\alpha}_{21}, \tilde{\alpha}_{22}$ & $v_{0} \leftrightarrow v_{1}$ \\
$\mathbf{p}_{31}$ & $\mathbf{p}_{41}$ & $v_{0} \leftrightarrow v_{1}$ \\
& & $\mathbf{p}_{20} \leftrightarrow \mathbf{p}_{60}, \mathbf{p}_{22} \leftrightarrow \mathbf{p}_{42}$, \\
& $\tilde{\mathbf{p}}_{21} \leftrightarrow \tilde{\mathbf{p}}_{51}, \tilde{\mathbf{p}}_{22} \leftrightarrow \tilde{\mathbf{p}}_{42}$ \\
$\tilde{\mathbf{p}}_{31}, \tilde{\mathbf{p}}_{41}, \tilde{\mathbf{p}}_{32}$ & $\mathbf{p}_{31}, \mathbf{p}_{41}, \mathbf{p}_{32}$ & $\begin{array}{l}\mathbf{p}_{i j} \leftrightarrow \tilde{\mathbf{p}}_{i j}, \mathbf{a}_{32} \leftrightarrow \tilde{\mathbf{a}}_{32} .\end{array}$
\end{tabular}

In Section 4, we will apply this local construction across sector boundaries.

\section{One piece per sector when $4 \leq n \leq 8$}

Given the reparametrization $\rho$ defined in Section 2.2, the 'sampling' operators $h^{8}$ and $h_{\diamond}^{8}$ and the local $C^{2}$ construction of the previous section, the construction of the $n$-sided surface cap $\mathbf{x}: \Delta \times\{1, \ldots, n\} \rightarrow \mathbb{R}^{3}$ is now very simple to implement. We start by creating an approximation $\overline{\mathbf{x}}^{i}:=h^{8}\left(\mathbf{g} \circ R^{i} \rho\right), i=1, \ldots, n$, of the cap, where $R^{i}$ rotates the reparameterization by $2 \pi / n$ to the $i$ th segment of the guide $g$. We initialize the BB-coefficients to the reparameterized boundary data along the $i$ th boundary segment, and to the guide approximation otherwise:

$$
\mathbf{x}_{j k}^{i}:= \begin{cases}\left(\mathbf{b}^{i} \circ \gamma\right)_{j k}, & j+k>5, \\ \overline{\mathbf{x}}_{j k}^{i}, & j+k \leq 5 .\end{cases}
$$

The cap is then by construction a $C^{2}$ prolongation of the $\gamma$ reparameterized boundary data. For each sector boundary, we set the auxiliary coefficients of (13): $\mathbf{a}_{40} \leftarrow \mathbf{x}_{40}^{i}, \mathbf{a}_{32} \leftarrow \mathbf{x}_{32}^{i}$ and $\tilde{\mathbf{a}}_{32} \leftarrow \mathbf{x}_{32}^{i+1}$; then the coefficients $\mathbf{x}_{j k}^{i}$ are updated according to the local construction of Section 3.

Examples of the construction are shown in Figure 8. We picked the multi-saddle, since it cannot be correctly resolved if a quadratic determines the surface where the patches meet [18]. (This supports the earlier claim of better shape control with our construction when the degree is 8). The con-

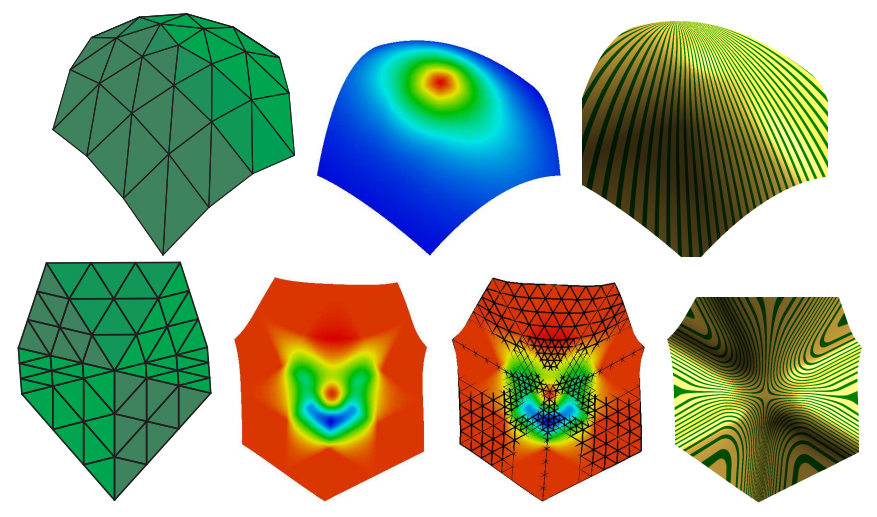

Figure 8: Moderate valence, $n<9$. (top) Elliptic 5-sided surface, (bottom) 7-sided monkey saddle. (left) input mesh; (middle) Gauss curvature shading; (right) highlight shading. The Gauss curvature, bottom, is shown additionally with the control net superimposed to verify that any abrupt changes of curvature occurs in the quartic input ring and is not due to the construction.

struction works well for valences $n=4,5,7,8$. Starting from valence 9 , we expected and detected shape problems due to the terms $\bar{c}:=1-c$ (that follow from (3) and converge to zero as

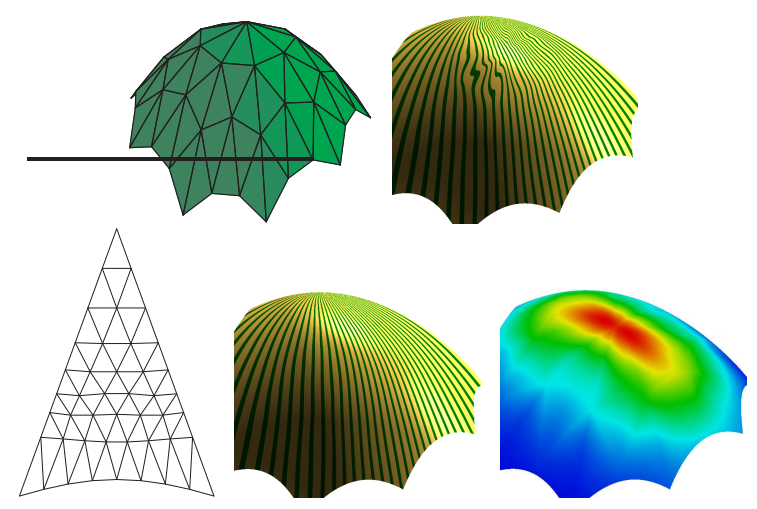

Figure 9: Single patch vs. 4-piece macro patch. (top, left) Input mesh, $n=9$ (top right) highlight lines of the blend based on a single polynomial piece of degree 8 per sector. (bottom, left) $\mathcal{F}_{5}$ applied to $\rho$. (bottom middle) Highlight lines of degree 8 4-piece macro patch and (right) corresponding Gauss curvature.
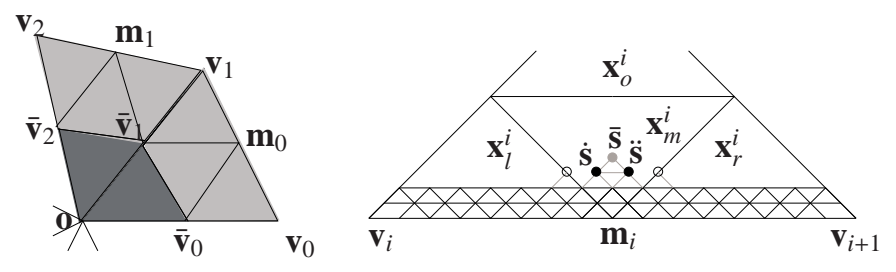

Figure 10: Macro-patch: (left) four polynomial pieces $\mathbf{x}_{o}^{i}, \mathbf{x}_{l}^{i}, \mathbf{x}_{m}^{i}, \mathbf{x}_{r}^{i}$ of degree 8 per sector $i$. (right) Subdivision and extension of the boundary data.

$n$ increases) in the denominator of $\gamma$ and hence the transformed data. Figure 9, middle left, shows the resulting slight fluctuations in the cap. Since the $\bar{c}$ terms result from the $C^{1}$ constraint (3), increasing the degree of $\mathbf{p}$ will not improve the situation. Instead, we now apply the spline paradigm.

\section{Four pieces per sector when $9 \leq n \leq 12$}

To obtain the improvement of Figure 9, bottom middle over Figure 9, top right, we use four patches of degree 8 per sector. The resulting extra spline ring reduces fluctuations, not by scaling as in mesh subdivision, but by finer sampling of the guide surface g. With

$$
\mathbf{m}_{i}:=\frac{1}{2}\left(\mathbf{v}_{i}+\mathbf{v}_{i+1}\right) \text { and } \overline{\mathbf{v}}_{i}:=\overline{\mathbf{c}} \mathbf{o}+\mathbf{c} \mathbf{v}_{i}
$$

each segment is split into four subtriangles as in Figure 10, left. That is the barycentric weights for the $C^{1}$ and the $C^{2}$ constraints are obtained from

$$
\begin{aligned}
\mathbf{m}_{1} & =\overline{\mathbf{v}}_{1}+\mathbf{v}_{1}-\mathbf{m}_{0}, \quad \mathbf{m}_{0}=\frac{1}{2 \mathrm{c}} \overline{\mathbf{v}}_{0}+\frac{1}{2 \mathrm{c}} \overline{\mathbf{v}}_{1}-\frac{\overline{\mathrm{c}}}{\mathrm{c}} \mathbf{o}, \\
\mathbf{v}_{1} & =\mathbf{m}_{0}+\frac{1}{2 \mathrm{c}}\left(\overline{\mathbf{v}}_{1}-\overline{\mathbf{v}}_{0}\right) .
\end{aligned}
$$

We now subdivide the boundary data to the three layers of coefficients shown in Figure 10, right and determine $h_{\diamond}^{8}$ at $\mathbf{o}$ and at the $\overline{\mathbf{v}}_{i}$. This again localizes the $C^{2}$ continuity constraints and we can solve according to Section 3 , except that we are missing three coefficients at $\mathbf{m}_{0}$. We set these coefficients with the 
help of the guide as follows. First we construct, by the same approach as in Section 2.2, a $C^{2}$ reparametrization macro-patch $\rho$ consisting of four pieces $q \in\{o, l, m, r\}$ per segment. Then we compute (see Figure 10, right) $\overline{\mathbf{x}}_{q}^{i}:=h^{8}\left(\mathbf{g} \circ R^{i} \rho_{q}\right)$ and set $\overline{\mathbf{s}}$ to the coefficient of $\overline{\mathbf{x}}_{m}^{i}$ indicated as grey disk, $\mathbf{a}_{1}$ to be the average of the coefficients of $\overline{\mathbf{x}}_{m}^{i}$ indicated by the black disks in Figure 10 , right, and $\dot{\mathbf{s}}:=\mathbf{a}_{1}+\mathbf{a}_{0}, \ddot{\mathbf{s}}:=\mathbf{a}_{1}-\mathbf{a}_{0}$ where

$$
\begin{aligned}
\mathbf{a}_{0}:= & \frac{c}{64}\left(c^{2}\left(\mathbf{p}_{10}-\mathbf{p}_{70}\right)+4 c^{2}\left(\mathbf{p}_{20}-\mathbf{p}_{60}\right)+5 c^{2}\left(\mathbf{p}_{30}-\mathbf{p}_{50}\right)\right. \\
& +c \bar{c}\left(\mathbf{p}_{01}-\mathbf{p}_{71}\right)+5 c \bar{c}\left(\mathbf{p}_{11}-\mathbf{p}_{61}\right) \\
& +9 \mathrm{c} \bar{c}\left(\mathbf{p}_{21}-\mathbf{p}_{51}\right)+5 c \bar{c}\left(\mathbf{p}_{31}-\mathbf{p}_{41}\right) \\
& \left.+\bar{c}^{2}\left(\mathbf{p}_{02}-\mathbf{p}_{62}\right)+4 \bar{c}^{2}\left(\mathbf{p}_{12}-\mathbf{p}_{52}\right)+5 \bar{c}^{2}\left(\mathbf{p}_{22}-\mathbf{p}_{42}\right)\right)
\end{aligned}
$$

The construction of $\rho$ is analogous. Figure 11 shows the result

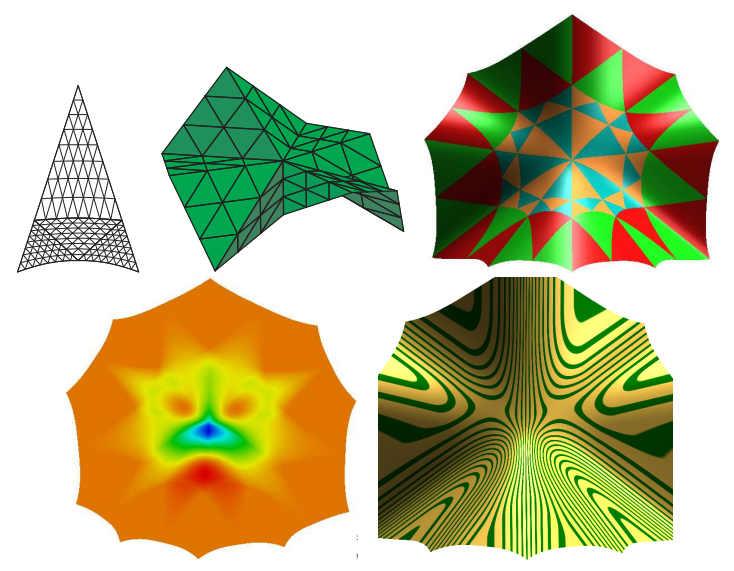

Figure 11: High valence. (top) Reparametrization for $n=10$, input mesh outlining a 10-sided fourfold saddle, subpatches. (bottom) Gauss curvature shading and highlight lines on the constructed surface.

for a higher-order saddle.

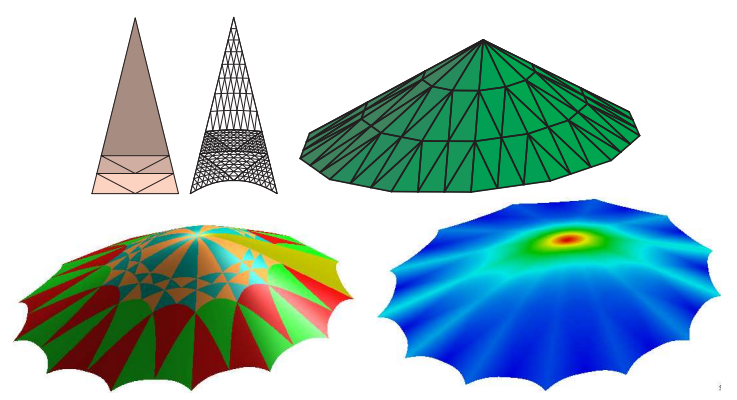

Figure 12: Very high valence. Reparametrization for $n=13$ and elliptic 13sided surface.

\section{High and low valences}

For valences still higher than 12, we repeat the macro-patch split, pinning down extra degrees of freedom by the guide surface. Without going into the technical details, the split of Figure 12, top left, handles even higher-valent input: the fluctuations of mean curvature in the input surface ring are extended

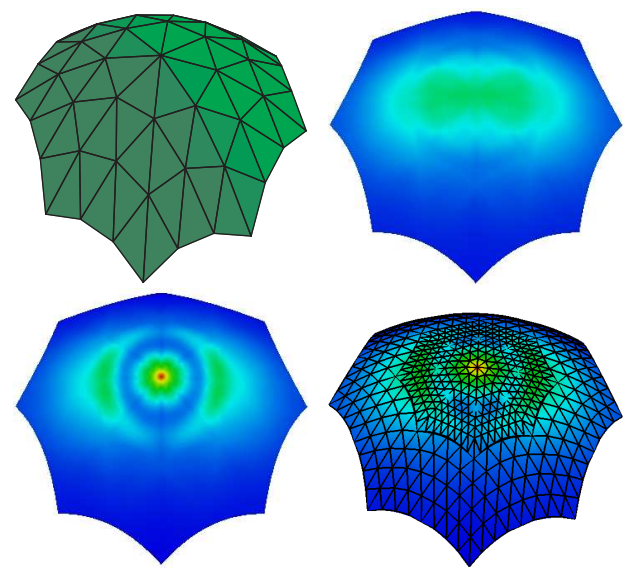

Figure 13: Shape problem of un-guided, linear functional-minimizing surface capping for higher valences. (top left) 8-sided elliptic input mesh; (top right) Gauss shading of guided cap; (bottom left) Gauss shading of cap obtained by minimizing $\mathcal{F}_{5}$ : the surface oscillates as is apparent on the (bottom right) where the surface reaches above its Bézier control net at 8 spots.

into the cap as is desirable, but the center is smooth. The whole procedure reminds of subdivision. Indeed, subdivision algorithms are known to do well at averaging out saddle data; but poorly when dealing with elliptic shapes as in Figure 12.

For low valences a simpler approach is possible. On the technical level, for $n=4$, the circulant system of $C^{2}$ constraints (8) of the guide offers two additional free coefficients, so that $\alpha_{32} \mathbf{a}_{32}+\tilde{\alpha}_{32} \tilde{\mathbf{a}}_{32}$ in the formula (15) for $\mathbf{p}_{32}$ can be replaced by $\left(\alpha_{32}+\tilde{\alpha}_{32}\right) \overline{\mathbf{p}}_{32}$, where $\overline{\mathbf{p}}_{32}$ is an unconstrained coefficient set by the guide surface. Guided surfacing is reliable also for $n=4$. But so is simply setting up the constraints and minimizing the functional $\mathcal{F}_{5}$ for each coordinate separately over the free points (circled in Figure 14, including the central coefficient $\mathbf{g}_{00}^{i}$ ). In fact, this approach generates well-shaped surfaces for valences up to $n=7$. However, Figure 13 shows the limitations of minimizing these geometrically unmotivated functionals for higher valences in ambient space. We observed such unwanted fluctuations for a wide sampling of (linear combinations of) functionals $\mathcal{F}_{m}$. This situation becomes worse when we need to use four pieces per macro-patch and the problem is even more underconstrained.

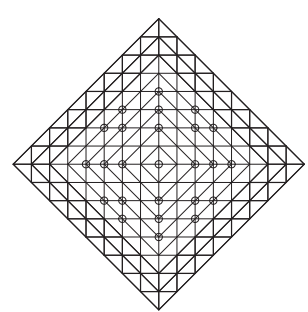

Figure 14: $C^{2}$ degree 8 construction for valence $n=4$. Outer lines indicate transformed boundary data. The coefficients indicated as circles are set by minimizing $\mathcal{F}_{5}$ for each coordinate separately.

Finally, if the object in question is not a tetrahedron or must have tetrahedral, rather than higher valent star-like spikes, vertices with $n=3$ are best removed from triangular layouts. Fig- 


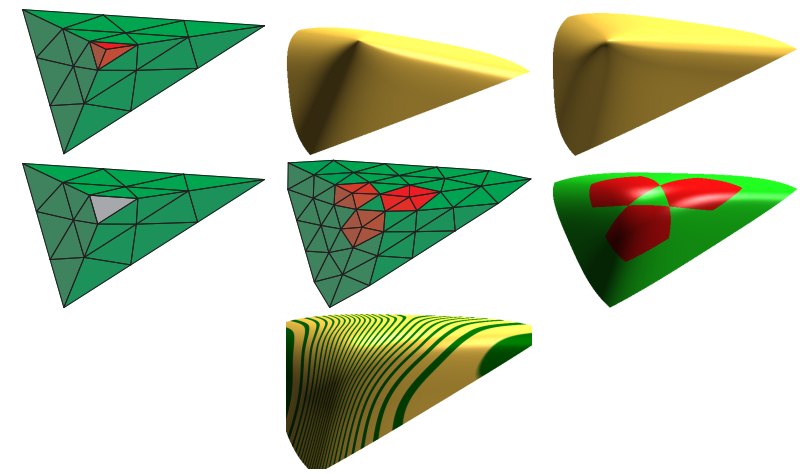

Figure 15: Valence $n=3$. top row: (left) elliptic input mesh; (middle) Localized Loop subdivision of the control net; (right) un-guided cap after one subdivision step. middle row: (left) $n=3$ node removed (middle) one Loop subdivision step applied since new 5-valenced nodes are not separated; (right) resulting surface; (bottom) with highlight lines.

ure 15 illustrates the effect of removal as well as the effect of one additional subdivision step applied to the input net.

Special treatment of high and of low valences has repeatedly been reported as the price for better surface quality. Here, with the exception of the structural change for $n=3$, all cases can be covered by guided surfacing.

Acknowledgement: This work was supported in part by NSF Grant CCF-0728797. We thank Georg Umlauf for feedback on an early version of the manuscript.

\section{References}

[1] Carl de Boor, Klaus Höllig, and Sherman Riemenschneider, Box splines, Springer-Verlag New York, Inc., New York, NY, USA, 1993.

[2] John A. Gregory and Jörg M. Hahn, $A C^{2}$ polygonal surface patch, Comput. Aided Geom. Design 6 (1989), no. 1, 69-75.

[3] John A. Gregory and Jianwei Zhou, Irregular $C^{2}$ surface construction using bi-polynomial rectangular patches, Comput. Aided Geom. Design 16 (1999), no. 5, 423-435. MR 1695665

[4] G. Greiner, Variational design and fairing of spline surfaces, Computer Graphics Forum 13 (1994), no. 3, C/143-C/154.

[5] C. M. Grimm and J. F. Hughes, Modeling surfaces of arbitrary topology using manifolds, Computer Graphics 29 (1995), no. Annual Conference Series, 359-368.

[6] Jörg Hahn, Filling polygonal holes with rectangular patches, Theory and practice of geometric modeling (Blaubeuren, 1988), Springer, Berlin, 1989, pp. 81-91. MR 91a:65032

[7] K. Karčiauskas and J. Peters, Concentric tesselation maps and curvature continuous guided surfaces, Computer-Aided Geometric Design 24 (2007), no. 2, 99-111.

[8] $\_$, Guided spline surfaces, Computer Aided Geometric Design (2009 N1), 1-20.

[9] M.J. Lai and P. Wenston, Bivariate splines for fluid flows, Computers and Fluids 33 (2004), 1047-1073.

[10] Charles Loop, Second order smoothness over extraordinary vertices, Symp Geom Processing, 2004, pp. 169-178.

[11] Charles T. Loop and Scott Schaefer, $G^{2}$ tensor product splines over extraordinary vertices, Comput. Graph. Forum 27 (2008), no. 5, 13731382.

[12] Henry P. Moreton and Carlo H. Séquin, Functional optimization for fair surface design, Computer Graphics 26 (1992), no. 2, 167-176.

[13] J. Cotrina Navau and N. Pla Garcia, Modeling surfaces from meshes of arbitrary topology, Comput. Aided Geom. Design 17 (2000), no. 7, 643671. MR 2001h:65022

[14] J. Peters, Curvature continuous spline surfaces over irregular meshes, Computer-Aided Geometric Design 13 (1996), no. 2, 101-131.
[15] H. Prautzsch, Freeform splines, Computer Aided Geometric Design 14 (1997), no. 3, 201-206.

[16] H. Prautzsch, W. Boehm, and M. Paluzny, Bézier and b-spline techniques, Springer Verlag, 2002.

[17] Hartmut Prautzsch and Georg Umlauf, Triangular $G^{2}$ splines, Curves and Surfaces, 1999, pp. 335-342.

[18] gree, ACM Transactions on Graphics 25 (2006), no. 4, 1281-1293.

[19] U. Reif, TURBS - topologically unrestricted rational B-splines, Constructive Approximation 14 (1998), no. 1, 57-77.

[20] William Welch and Andrew Witkin, Variational surface modeling, Computer Graphics (SIGGRAPH '92 Proceedings) 26 (1992), no. 2, 157-166.

[21] Xiuzi Ye, Curvature continuous interpolation of curve meshes, Computer Aided Geometric Design 14 (1997), no. 2, 169-190.

\section{Appendix: A default guide surface construction}

In the absence of a user-provided guide, we construct a $C^{2}$ piecewise polynomial map $\mathbf{g}$ of total degree 5 . While there is no restriction on the degree of the guide surface, in our experience guide surfaces consisting of $n C^{2}$-connected triangular patches of total degree 5 suffice to approximate boundary data well and there are no noticeable improvements in the final surface if the degree is higher.

\subsection{Setting the degrees of freedom to match boundary data}

By elementary computations of the $C^{1}$ and $C^{2}$ constraints for the layers $\mathbf{g}_{j k}$ for $j+k \in\{4,5\}$ and Lemma 1 , the free coefficients of $\mathbf{g}$ are (shown as black bullets in Figure 5)

$$
\mathbf{g}_{j k}^{*}: \quad \mathbf{g}_{j k}^{0}, j+k \leq 2, \quad \mathbf{g}_{j 0}^{i}, j=3,4,5, \quad \mathbf{g}_{22}^{i}, \mathbf{g}_{23}^{i}, \mathbf{g}_{32}^{i} .
$$

We fix the central coefficient as an average of the central mesh point $\mathbf{c}_{0}$ and the first ring of input mesh points $\mathbf{c}_{i}$ (Figure 1) as

$$
\begin{aligned}
\mathbf{g}_{00}^{i} & :=\beta \mathbf{c}_{0}+\frac{1-\beta}{n} \sum_{j=1}^{n} \mathbf{c}_{j}, \\
\beta & :=\frac{3}{11-8 \eta}, \eta:=\frac{1}{2}\left(\frac{3}{8}+\frac{(3+2 \mathrm{c})^{2}}{64}\right)+\frac{1}{2} \frac{1+\mathrm{c}^{2}}{2} .
\end{aligned}
$$

Here $\eta$ was determined by experiment. Given the boundary data b and the characteristic ring $\chi$ of Loop subdivision, we can now determine the free points $\mathbf{g}_{j k}^{*}$ by best matching guide and data. For each coordinate, separately, we minimize

$$
\min _{\mathbf{g}_{j k}^{*}}\left\|h_{\diamond}^{8}(\mathbf{g} \circ \chi)-h_{\diamond}^{8}(\mathbf{b})\right\|_{2}^{2},
$$

i.e. we minimize over all sectors the squared distances between the coefficients obtained from sampling the reparameterized guide, $h_{\diamond}^{8}(\mathbf{g} \circ \chi)$, and the degree-raised boundary data as indicated in Figure 16, right.

Acknowledgement. This work was supported by the National Science Foundation Grant 0728797. 

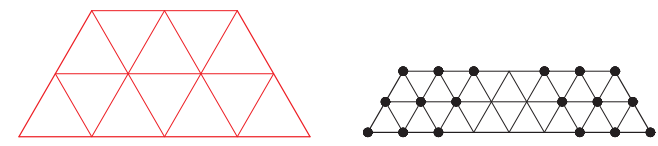

Figure 16: Guide construction. (left) One segment of the boundary data, b, (right) raised to degree 8. The points marked as bullets are the output of $h_{\diamond}^{8}(\mathbf{b})$ resulting from raising the degree of $\mathbf{b}$ to eight and selecting the points. They are approximated by the structurally identical output of $h_{\diamond}^{8}$ applied to the reparameterized guide: $h_{\diamond}^{8}(\mathbf{g} \circ \chi)$. 